(2) Open Access Full Text Article

\title{
Wearing facemasks when performing lumbar punctures: a snapshot of current practice amongst trainee doctors
}

This article was published in the following Dove Press journal:

Local and Regional Anesthesia

19 November 2010

Number of times this article has been viewed

\author{
Rajiv Malhotra' \\ Sara Kelly ${ }^{2}$ \\ 'Weston General Hospital, \\ Weston-super-Mare, Somerset; \\ ${ }^{2}$ University Hospital Coventry \\ and Warwickshire, Coventry, UK
}

Correspondence: Rajiv Malhotra 33 Stowe Close, Liverpool, L25 7YE, UK $\mathrm{Tel}+4407931984456$

Fax +44079 31984456

Email rajivmalhotral@hotmail.com
Purpose: Infective complications of lumbar puncture are not common, but are a significant source of mortality. Causative pathogens have been traced to the oropharynx of the operator, and it is likely that wearing facemasks will minimize the risk of iatrogenic meningitis. The aim of this survey was to assess whether doctors currently wear facemasks when performing lumbar punctures.

Methods: We constructed an anonymous survey asking about the use of a facemask when performing lumbar punctures. This was distributed to trainee doctors in medical specialties at the West Midlands and Severn Deaneries in the UK.

Results: The response rate was $72 \%(72 / 100)$. Responders had performed, on average, a total of 15 (range 3-22) lumbar punctures. Only 27 of the doctors (37.5\%) wore a facemask when performing lumbar punctures. CT 1-2 doctors were five times more likely than registrars to wear a facemask ( $53 \%$ versus $10 \%$ ). Similarly, the likelihood of wearing a facemask decreased with the number of times the procedure had been performed.

Discussion: There are varying practices regarding the use of facemasks for lumbar punctures amongst doctors, with significant differences according to grade and level of experience. Facemasks should be used as part of a "maximal sterile precautions" approach to reduce the risk of infective complications.

Keywords: infection control, iatrogenic meningitis, facemasks

\section{Introduction}

Infective complications of lumbar puncture are not common, but are a significant source of morbidity and mortality. Iatrogenic meningitis is estimated to occur in one in every 5000 patients who undergo dural puncture. ${ }^{1}$ There are no explicit guidelines covering the appropriate infection control measures to take when performing lumbar punctures.

The most common causative organisms are streptococcal species, followed by Gram negative organisms, such as Pseudomonas aeruginosa. The exact mechanism by which these infective complications occur has not been conclusively identified. However, bacterial isolates from patients with meningitis postdural puncture have been molecularly matched to strains obtained from the oropharynx of doctors who performed the procedure. ${ }^{1,2}$ Therefore, the use of face masks may help to prevent iatrogenic infection during the procedure. The wearing of a face mask, as part of full barrier protection, has been shown to reduce the risk of central venous line-associated infections. ${ }^{3}$ This survey aims to assess whether trainee doctors wear a facemask when performing lumbar punctures. 


\section{Materials and methods}

A total of 188 doctors were given an anonymous survey asking about specific infection control measures they undertake when performing a lumbar puncture. The doctors were all trainees in medical specialties, across various hospitals within the Severn and West Midlands deaneries in the UK. The questionnaire is shown in Figure 1.

\section{Results}

The response rate was $72 \%$ ( 72 of 100), consisting of $30 \mathrm{CT}$ 1-2 doctors, 22 ST 3-7 doctors, and 20 registrars. Responders had performed, on average, a total of 15 (range 3-22) lumbar punctures. Only 27 of the doctors (37.5\%) wore a facemask when performing lumbar punctures (16 CT 1-2 doctors, nine ST 3-7 doctors, two registrars). CT 1-2 doctors were five times more likely than registrars to wear a facemask (16/30 versus $2 / 20$, Figure 2). Similarly, the likelihood of wearing a facemask decreased with the number of times the procedure had been performed (Figure 3).

\section{Discussion}

The risk of infective complications after a lumbar puncture is low, but one study showed that the mortality rate in those with iatrogenic meningitis was $36 \% .{ }^{2}$ There is no consensus on the "gold standard" infection control measures to be taken by doctors when performing lumbar punctures.

A great deal of evidence supporting the use of "maximal sterile precautions" in central venous line insertion has been generated..$^{3-5}$ This approach has been shown to reduce significantly the risk of central venous catheter-related infections, ${ }^{4,5}$ although the impact of wearing a facemask by itself has not been investigated. Whilst this procedure involves a foreign object remaining in the patient, it is likely that such measures would improve patient safety.

Given that postdural puncture infections are rare, it is highly significant that there are reports of clustering of cases, suggesting that the operator has a role to play in the etiology.

A survey on the use of facemasks when performing a lumbar puncture.
1. What grade of doctor are you? (Please circle)
CT 1-2 ST 3-7 Registrar
2. How many lumbar punctures have you performed in your career so far?
3. Do you wear a facemask when performing a lumbar puncture? Y/N
Thank you for your response. All questionnaires are kept anonymous.

Figure I Questionnaire distributed to doctors.

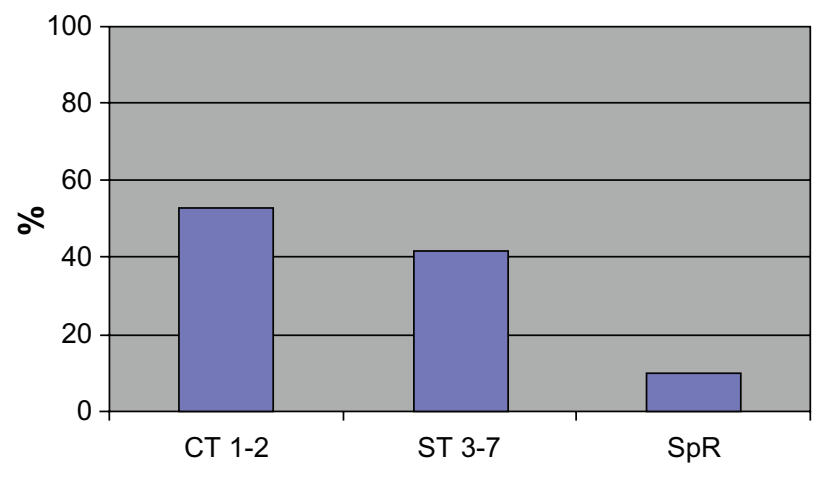

Figure 2 Percentage of each grade of trainee doctors who wear a facemask when performing a lumbar puncture.

Most of the organisms causing iatrogenic meningitis after lumbar puncture are mouth commensals, and the "droplet theory" implicates aerosolized organisms from the upper airways of doctors. Indeed, Trautman et $\mathrm{al}^{6}$ report a case of Staphylococcus aureus meningitis in which the organism matched the nasal swabs of the operator, and none of the other nearby staff. Such evidence would support the concept that wearing facemasks could reduce the risk of infective complications. Despite there being evidence that facemasks reduce bacterial contamination of a patient or operative field, ${ }^{7,8}$ there is no evidence that their use is associated with a reduction in patient infections (eg, surgical site infections). ${ }^{9}$

This survey shows that most doctors do not use facemasks when performing lumbar punctures. This could be for several reasons. Firstly, the incidence of infective complications is low and the awareness of this potential complication may be limited. Secondly, there may be a lack of availability of facemasks on wards. Thirdly, there may be a lack of perceived evidence behind the use of facemasks to reduce iatrogenic infections. There is no conclusive evidence that the use of facemasks specifically reduces the incidence of iatrogenic infections. Indeed, in a questionnaire-based

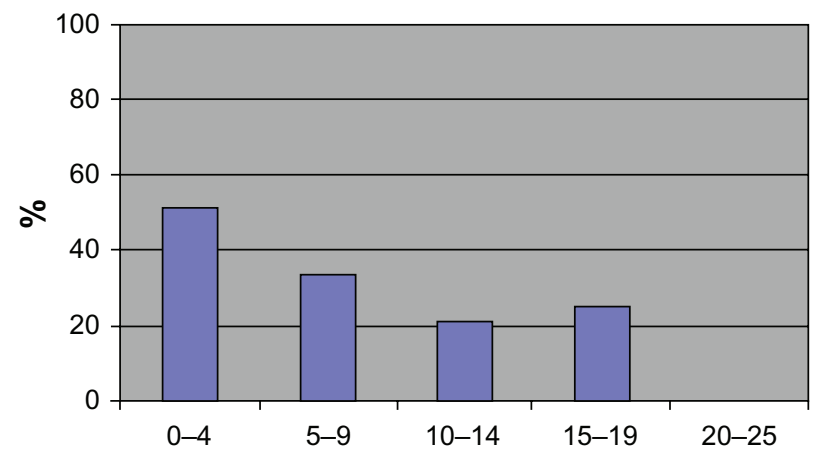

Figure 3 Percentage of trainee doctors who wear a facemask when performing a lumbar puncture according to the number of lumbar punctures they have performed. 
survey by Erasmus et $\mathrm{al}^{10}$ doctors cited a lack of evidence as the reason for poor compliance with hand washing in the intensive care setting.

We also show that the wearing of facemasks dropped with both the seniority of the doctor and experience in performing lumbar punctures. Richman et al also showed that junior doctors were more likely than senior doctors to wear gloves during invasive procedures in children (94\% versus $46 \%$ ). ${ }^{11}$ It is likely that improved education in medical school contributes to this. However, the lack of compliance by senior doctors is a worrying trend because it has been shown that the infection control measures of junior staff are influenced by their seniors; junior staff members were less likely to wash their hands (odds ratio 0.2) if an accompanying senior staff member did not wash their hands. ${ }^{12}$

There are varying practices regarding aseptic technique for lumbar punctures amongst doctors, with significant differences according to grade and level of experience. There is theoretic evidence that facemasks would reduce contamination of the patient, but no conclusive evidence that their use reduces the incidence of iatrogenic infections after lumbar puncture. However, we believe that facemasks should be used as part of a "maximal sterile precautions" approach with this invasive procedure.

\section{Disclosure}

The authors report no conflicts of interest in this work.

\section{References}

1. Schneeberger PM, Janssen M, Voss A. Alpha-hemolytic streptococci: A major pathogen of iatrogenic meningitis following lumbar puncture. Case reports and a review of the literature. Infection. 1996;24(1):29-33.

2. Baer ET. Post-dural puncture bacterial meningitis. Anesthesiology. 2006;105(2):381-393.

3. Berenholtz SM, Pronovost PJ, Lipsett PA, et al. Eliminating catheterrelated bloodstream infections in the intensive care unit. Crit Care Med. 2004;32(10):2014-2020.

4. Pearson ML. Hospital Infection Control Practices Advisory Committee Guidelines for prevention of intravascular device related infections. Infect Control Hosp Epidemiol. 1994;15:227-230.

5. Raad II, Hohn DC, Gilbreath BJ, et al. Prevention of central venous catheter-related infections by using maximal sterile barrier precautions during insertion. Infect Control Hosp Epidemiol. 1994; 15(4 Pt 1):231-238.

6. Trautman M, Lepper PM, Schmiyz FJ. Three cases of bacterial meningitis after spinal and epidural anesthesia. J Clin Microbiol Infect Dis. 2002;21(1):43-45.

7. Berger SA, Kramer M, Nagar H, Finkelstein A, Frimmerman A, Miller HI. Effect of surgical mask position on bacterial contamination of the operative field. J Hosp Infect. 1993;23(1):51-54.

8. McLure HA, Talboys CA, Yentis SM, Azadian BS. Surgical face masks and downward dispersal of bacteria. Anaesthesia. 1998;53(7): 624-626.

9. Lipp A, Edwards P. Disposable surgical face masks for preventing surgical wound infection in clean surgery. Cochrane Database Syst Rev. 2002;(1):CD002929.

10. Erasmus V, Brouwer W, van Beeck EF, et al. A qualitative exploration of reasons for poor hand hygiene among hospital workers: Lack of positive role models and of convincing evidence that hand hygiene prevents cross-infection. Infect Control Hosp Epidemiol. 2009;30(5):415-419.

11. Richman G, Dorsey A, Stayer S, Schwartz R. Compliance with standard precautions among pediatric anesthesia providers. The Internet Journal of Anesthesiology. 2000:4(4).

12. Lankford MG, Zembower TR, Trick WE, Hacek DM, Noskin GA, Peterson LR. Influence of role models and hospital design on hand hygiene of healthcare workers. Emerg Infect Dis. 2003;9(2):217-223.
Local and Regional Anesthesia

\section{Publish your work in this journal}

Local and Regional Anesthesia is an international, peer-reviewed, open access journal publishing on the development, pharmacology, delivery and targeting and clinical use of local and regional anesthetics and analgesics. The journal welcomes submitted papers covering original research, basic science, clinical studies, reviews \& evaluations,

\section{Dovepress}

guidelines, expert opinion and commentary, case reports and extended reports. The manuscript management system is completely online and includes a very quick and fair peer-review system, which is all easy to use. Visit http://www.dovepress.com/testimonials.php to read real quotes from published authors. 